\title{
MAMA TRÁNSITO AMAGUAÑA Y LA PUCE: "HUMANAMENTE IGUALES, CULTURALMENTE DIFERENTES”
}

\author{
MAMA TRÁNSITO AMAGUAÑA AND THE PUCE: “HUMANALLY THE SAME, CULTURALLY \\ DIFFERENT"
}
MAMA TRÁNSITO AMAGUAÑA E LA PUCE: “HUMANAMENTE O MESMO, CULTURALMENTE DIFERENTE"

\section{Jacqueline Elizabeth Cevallos Salazar', Alexandro Vinicio Cruz Mariño², Ruth Elizabeth Jimbo Sotomayor ${ }^{3}$, Irma del Carmen Gómez Walfandery ${ }^{4}$, José Julio Agualongo Tenelema ${ }^{5}$}

\author{
${ }^{1}$ Pontificia Universidad Católica del Ecuador, Ecuador, jcevallos498@puce.edu.ec \\ ${ }^{2}$ Pontificia Universidad Católica del Ecuador, Ecuador, avcruz@puce.edu.ec \\ ${ }^{3}$ Pontificia Universidad Católica del Ecuador, Ecuador, rejimbo@puce.edu.ec \\ ${ }^{4}$ Unidad Educativa Intercultural Bilingüe Tránsito Amaguaña, Ecuador, transitoamaguaa@yahoo.com \\ ${ }^{5}$ Unidad Educativa Intercultural Bilingüe Tránsito Amaguaña, Ecuador, warankito15@yahoo.com
}

\section{RESUMEN}

La formación médica combina procesos de aprendizaje teórico-prácticos que van de la mano con el servicio a la comunidad. Este vínculo impacta en el desarrollo académico de estudiantes y docentes con una comprensión más profunda de la importancia de la medicina integral que abraza el respeto al ser humano, su entorno, su lengua y su cultura. El presente trabajo presenta la experiencia del proyecto AprendizajeServicio de estudiantes de la carrera de medicina de la Pontifica Universidad Católica del Ecuador en la Unidad Educativa Intercultural Bilingüe Tránsito Amaguaña, un proceso que promueve la inclusión y el servicio médico en el margen de la aceptación de la diversidad cultural y la erradicación del racismo.

Palabras Claves: aprendizaje-servicio; educación de pregrado en medicina; cultura indígena; racismo.

\begin{abstract}
Medical training combines theoretical-practical learning processes gaining hands-on experience with community service; this link impacts academic developmen t of students and teachers with a deeper understanding of the importance of integral medicine that embraces respect for the human being, his environment, his language and his culture. This paper presents the experience of the Service-Learning project of students of the medical career of the Pontifical Catholic University of Ecuador in the Tránsito Amaguaña Intercultural Bilingual Educational Unit, a process that promotes inclusion and medical service in the margin of acceptance of the cultural diversity and the eradication of racism.
\end{abstract}

Key words: service-learning; undergraduate medical education; culture indigenous; racism. 


\section{RESUMO}

A formação médica combina processos de aprendizagem teórico-práticos que andam de mãos dadas com o serviço comunitário. Esta aliança impacta o desenvolvimento acadêmico de alunos e professores, com uma compreensão mais profunda da importância da medicina integral que abraça o respeito ao ser humano, seu meio ambiente, sua linguagem e sua cultura. Este artigo apresenta a experiência do projeto Service-Learning de alunos da carreira médica da Pontifícia Universidade Católica do Equador na Unidade Educacional Intercultural Bilíngue Trânsito Amaguaña, um processo que promove a inclusão e o atendimento médico além da diversidade cultural e erradicação do racismo.

Palavras chaves: aprendizagem-serviço; educação de graduação em medicina; culturas indígenas; racismo.

\section{1.- INTRODUCCION}

La migración del campo a la ciudad es uno de los problemas vigentes en el mundo y la región, según la Organización Mundial sobre Migraciones (OIM), las personas se movilizan de las zonas rurales a las zonas urbanas promovidos por la pobreza, la desigualdad, la inestabilidad política, desastres naturales y el cambio climático. Ecuador no es ajeno a esta realidad y cientos de pobladores originarios de las comunidades Kichwas de las provincias de Chimborazo, Tungurahua, Bolívar, Cotopaxi e Imbabura buscan mejorar sus ingresos con el traslado de familias indígenas a las grandes ciudades (Organización Mundial sobre Migraciones, 2015; Observatorio de Desplazamiento Interno, 2020).

De acuerdo al último censo de población del país, el $7.03 \%$ de los ecuatorianos se autoidentifica como indígena, $21.47 \%$ de su población vive en las grandes ciudades. Dentro de su colectivo presentan un mayor porcentaje de niños, niñas y adolescentes que el resto de las etnias del país. Ecuador reconoce a los menores como parte de los grupos de atención prioritaria, derecho por el cual el Estado garantiza salud, educación, vivienda, recreación, deporte y libertad de expresión (Asamblea Constituyente, 2008; INEC, 2010). Si bien no se conoce el desarrollo de la educación fuera de sus lugares de origen, está descrito que el área urbana posee los servicios y oportunidades que el área rural carece, condición que extiende la brecha social entre el campo y la ciudad, pero en la estadística general solo el
$66.55 \%$ de la población indígena logra obtener la educación básica y menos del $20 \%$ la educación media, lo que indica la falta de equidad incluso en las ciudades (Consejo Nacional para la Igualdad de Pueblos y Nacionalidades , 2020; Schoenfeld, 2010).

Desde una perspectiva interna, para las familias migrantes la continuación de la educación de los hijos presenta muchas barreras. Pocos menores consiguen insertarse en la educación pública, muchos desertan esperando el siguiente año lectivo y otros pasan a las arcas del trabajo infantil dentro y fuera de los mercados, lugares de acopio con mayor oferta para el trabajo indígena (Rodríguez Cruz, 2018). Conforme con el Instituto Nacional de Estadísticas y Censos del Ecuador (INEC), el 8.6\% de los niños, niñas y adolescentes entre los 5 y 17 años de edad trabaja, la cifra por etnia establece que el mayor porcentaje de niños trabajadores corresponde al pueblo indígena, con el 29\% (INEC, 2015).

Frente a esta realidad, desde 1990 un grupo de dirigentes profesionales indígenas y no indígenas vinculados al paradigma educativo intercultural, asumen levantar un proceso de revitalización pedagógico mediante la creación de la Unidad Educativa Intercultural Bilingüe “Tránsito Amaguaña”, entidad que vela por garantizar una educación bilingüe kichwa-castellano para niños, niñas y jóvenes indígenas del Mercado Mayorista, al Sur de la ciudad de Quito (UEIB Tránsito Amaguaña, 2019). 
El Sistema de Educación Intercultural Bilingüe (SEIB), en concordancia con la Constitución del Ecuador, tiene como objetivo fortalecer la construcción de un país multilingüe y plurinacional, lo que ofrece a las 14 nacionalidades y a todos los pueblos del territorio el derecho a contar con la mejor educación en su lengua materna y bajo su cultura. En este margen, la Unidad Intercultural Bilingüe “Tránsito Amaguaña” es el espacio educativo creado para la formación integral de los niños, niñas y jóvenes indígenas migrantes con afirmación de su identidad cultural y lengua. De esta forma, estudiantes, docentes y familias caminan juntos en busca del Sumak Kawsay o Buen Vivir (Asamblea Constituyente, 2008; Ministerio de Educación del Ecuador, 2015).

Si bien la Unidad “Tránsito Amaguaña” es autosustentable, confronta continuamente condiciones adversas en temas de recursos e inclusión. Su modelo educativo en la ciudad se desarrolla con el requerimiento de servicios externos, entre ellos la salud, es donde se entrelaza la Pontificia Universidad Católica del Ecuador (PUCE) que desde la vinculación con la comunidad y las prácticas preprofesionales trabaja de forma mancomunada en el servicio social (Pontificia Universidad Católica, 2019).

La Facultad de Medicina de la PUCE, tiene como objetivo de grado formar médicos con altos estándares científicos y con un vínculo estrecho del estudiante con la comunidad, en armonía con la Atención Primaria en Salud, que se concibe como la estrategia para satisfacer soluciones a los problemas de salud de los pueblos. En este contexto el estudiante adquiere un panorama global de la salud que incidirá de manera positiva en su formación médica holística, integral e integrada (Consejo Académico de la Facultad de Medicina de la Pontificia Universidad Católica del Ecuador, 1994).

La esencia de la carrera de medicina consiste en unificar los conocimientos teóricos, prácticos y éticos, y lo consigue a través de la asignatura conocida como IDIS, de sus siglas: Integración de la Docencia y la Investiga- ción aplicada al Servicio, una metodología innovadora en cuanto a la vinculación y compromiso del estudiante con la comunidad bajo la tutoría de docentes especialistas en Medicina Familiar. La concepción del proyecto nace en la aspiración de generar en los estudiantes de medicina destrezas como la comunicación acertada, el respeto a la interculturalidad, la compresión de la cosmovisión de los pueblos indígenas y su lengua, sumado a la atención médica del programa de la salud escolar, habilidades ajustadas al perfil de egreso de la carrera y dentro del margen del Modelo de Atención Integral de Salud del Ecuador (MAIS) (Ministerio de Salud Pública del Ecuador, 2018).

Con estos antecedentes, ambas instituciones se asocian en un espacio único para compartir y aprender. El proyecto se inscribió como Mama Tránsito y la PUCE, por un lado, se genera el conocimiento del servicio intercultural (entre culturas) en el estudiante de medicina, y por otro, se oferta un servicio cálido de salud desde el equipo del IDIS-PUCE a los estudiantes, familias y docentes de la unidad educativa. En síntesis, a través de la metodología de Aprendizaje-Servicio, se compuso un espacio con el intercambio continuo de saberes y destrezas, un intercambio interdisciplinario y biopsicosocial.

\section{2.- SERVICIO PRESTADO}

Se presentó el proyecto a las autoridades de la unidad educativa con la oferta de una atención médica bajo los componentes de la salud escolar. La idea fue brindar una atención profesional con óptica intercultural y acople al paradigma de Mama Tránsito Amaguaña. De esta forma, durante los períodos académicos 2018-01 al 2019-02, los diferentes grupos de IDIS comunitario de segundo, tercero y quinto nivel de la Facultad de Medicina, junto a su médico tutor, ofertaron el servicio a los estudiantes de Educación General Básica y del Bachillerato General Unificado, a sus familias y docentes de la institución. 
La salud escolar de forma general brinda atención médica dentro de los diferentes establecimientos educativos, desde la educación inicial al bachillerato. El objetivo es proporcionar el acceso a la salud integral en el entorno escolar, con la detección temprana y oportuna de problemas de salud y la identificación de factores de riesgo.

En este sentido, los equipos brindaron estrategias de promoción de la salud, prevención de la enfermedad, diagnóstico, tratamiento, acompañamiento y derivación en el margen del MAIS, lo que generó un espacio de interacción y convivencia con alrededor de 100 estudiantes y su planta docente.

Las actividades ofertadas por grupo etario de escolares $\mathrm{y}$ adolescentes se resumen en:

- Consulta de atención médica integral.

- Antropometría, screening visual, screening auditivo, campañas de vacunación, desparasitación, programa de micronutrientes.

- Actividades de promoción y prevención como: charlas, talleres, conferencias, casas abiertas.

- Espacios de actividades lúdicas.

\section{3.- APRENDIZAJES PROPUESTOS Y LOGRADOS}

La cultura comprende los saberes, valores, prácticas y principios que orientan a un grupo social; el reconocimiento cultural constituye la interrelación de las personas en medio de la diversidad. Para construir la interculturalidad, es necesario vivir el contraste entre el conocimiento occidental y la sabiduría de los pueblos andinos (Álvarez \& Montaluisa, 2017). Durante los diferentes períodos, estudiantes de medicina y sus tutores observaron y aprendieron del idioma kichwa, las costumbres y la medicina tradicional, lo que forja un principio de servicio holístico ante la riqueza cultural del país.

El acercamiento y trabajo directo con los actores de la unidad generó un análisis profundo sobre los determinantes sociales y de salud de la población indígena. De esta forma los estudiantes de medicina vivificaron esta problemática, lo que construyó un espacio de reflexión sobre temas de derecho, acceso, determinantes de la salud, vulnerabilidad y discriminación, objetivos de la asignatura IDIS.

La educación fomenta empatía y tolerancia entre las personas, contribuye a crear sociedades y ambientes más pacíficos y saludables. El proyecto Mama Tránsito y la PUCE permitió la interrelación de la educación bilingüe intercultural y la academia universitaria lo que significa un espacio de crecimiento para todos los actores: estudiantes de ambas instituciones, docentes interculturales y docentes universitarios.

Por otra parte, la salud se define como un "estado de completo bienestar físico, mental y social", que depende de los contextos económicos, sociales, políticos, culturales y ambientales en el que se desarrollan las personas (Organización Mundial de la Salud, 2021). Los estudiantes y el personal de "Tránsito Amaguaña" compartieron de la Atención Primaria en Salud, al participar activamente del programa, lo que además llevó a comprender que la salud integral debe cubrir las diferentes necesidades sean de índole afectivo, cultural, social y sanitario; este servicio debe ser ofertado por todo sistema sanitario en el marco de los derechos sociales. Si las actividades de la PUCE generaron un ambiente participativo de atención intercultural, esta experiencia puede ser extrapolada a otros espacios.

\section{4.- DIFICULTADES ENCONTRADAS}

El presupuesto es un limitante en todo proyecto. En la educación, al igual que en la salud, los insumos y requerimientos son permanentes.

Por otra parte, la resolución del cuadro clínico a veces depende de la prontitud de respuesta por parte del sistema de salud, la demora en la referencia y contrareferencia hacia y desde otros niveles dificulta que el 
estudiante puede dar el seguimiento adecuado a los pacientes. A su vez, el tiempo de rotación del IDIS en la comunidad es corto para vislumbrar el impacto de las intervenciones ofertadas y la resolución integral de problemas médicos.

Pese a estas dificultades, estudiantes y docentes de ambas instituciones se apoyaron en un intercambio recíproco cultural que se conoce como "ranti ranti", dar y recibir.

\section{5.- CONCLUSIONES Y REFLEXIONES}

La Constitución del Ecuador consagra la salud como un derecho humano fundamental y garantiza suministrar a todas las personas calidad de vida lo que confiere el acceso universal a la salud, esto incluye la igualdad de atención a los pueblos y nacionalidades ancestrales. La Ley Orgánica de Salud en su artículo 25 promueve y respeta el conocimiento y prácticas tradicionales de los pueblos indígenas, montubios y afroecuatorianos, lo que rompe la hegemonía del enfoque occidental e incorpora el enfoque intercultural a todos los servicios del Estado, preceptos que deben ser incluidos en la formación de los futuros médicos (Congreso Nacional, 2015).

En el margen del derecho a la igualdad de oportunidades para niños, niñas y adolescentes, la etnia indígena y todas las etnias deben tener acceso a un servicio de calidad y calidez, con profundo respeto a su cosmovisión y con los más altos estándares de la atención médica.

Es evidente que la participación activa del estudiante de medicina en campo puede convertirse en una experiencia positiva tanto en su formación científicotécnica, así como en la concientización de la no discriminación que van de la mano de los principios éticos, la empatía y los valores, parte de las competencias del saber ser del médico.

El Aprendizaje-Servicio es uno de los mecanismos pedagógicos para disminuir las brechas sociales y conse- guir la convivencia entre diferentes etnias, pueblos y lenguas al conectar la academia con el territorio (Chiva-Bartoll et al., 2018). En el proyecto Mama Tránsito y la PUCE, la comunión de la cultura indígena y la medicina ha permitido el enriquecimiento mutuo, con la fusión de diferentes saberes y la construcción de un nuevo significado de la medicina integral en los estudiantes; queremos médicos integrales, los formamos en espacios interculturales.

Pese a las diferentes adversidades, confiamos que al convertir una dificultad en una oportunidad, los estudiantes de medicina puedan comprender la lucha por la sostenibilidad de la estrategia de la Atención Primaria en Salud, sobre todo en el primer nivel de atención, al reflejar con su accionar futuras soluciones que promuevan un sistema de salud más equitativo.

Rosa Elena Amaguaña Alba, conocida como Mama Tránsito Amaguaña, fue una activista indígena que luchó por la equidad social de los pueblos del Ecuador. Junto con Dolores Cacuango organizó las primeras escuelas bilingües indígenas, escuelas clandestinas al no ser reconocidas por los gobiernos de turno. Una de sus frases más recordadas dice: "Así unidos, mezcladitos, como trigo y quinua, mezcladitos", palabras que hacen insignia al crecimiento en el campo de la quinua americana y del trigo europeo, así crecemos todos, humanamente iguales y culturalmente diferentes, pero juntos en la lucha por la gran minga de la vida.

\section{Referencias bibliográficas}

Álvarez, C., \& Montaluisa, L. (2017). Perfiles de las lenguas y saberes del Ecuador (IICSAE (ed.); Primera). La Guaragua. https://doi.org/8.242.217.84:8080

Chiva-Bartoll, Ó., Pallarès-Pique, M., Aguilar, J., Millán, A., Traver, J., Sales, A., Moliner, O., Rivera, E., Sánchez, M., Giles, J., Gil-Gómez, J., \& Felix, S. (2018). Aprendizaje-servicio: pasaporte para un futuro mejor - Dialnet (Primera). Egregius. https://dialnet.unirioja. es/servlet/libro? codigo $=711095$ 
Congreso Nacional. (2015). Ley Orgánica Del Sistema Nacional De Salud. https://doi.org/Registro Oficial Suplemento \# 423

Consejo Académico de la Facultad de Medicina de la Pontificia Universidad Católica del Ecuador. (1994). Creación de la Facultad de Medicina. 1-7.

Consejo Nacional para la Igualdad de Pueblos y Nacionalidades, \& (CNIPN). (2020). Agenda para la igualdad de derechos de las Nacionalidades y Pueblos Indígenas, Pueblo Afroecuatoriano y Pueblo Montubio 20192021. Manthra Comunicación. http://www.pueblosynacionalidades.gob.ec/wp-content/uploads/2020/02/ Agenda-Nacional-para-la-Igualdad-de-Pueblos-y-Nacionalidades.pdf

Constituyente, A. (2008). Constitución de la República del Ecuador (2008). https://www.acnur.org/fileadmin/ Documentos/BDL/2008/6716.pdf

INEC. (2010). Estadísticas de etnicidad en censos, encuestas de hogares y registros administrativos. https:// www.inei.gob.pe/media/dme/17.MarleneHaro.pdf

INEC, I. N. de E. y C. (2015). Trabajo infantil en Ecuador: Hacia un entendimiento integral de la problemática. https://www.ecuadorencifras.gob.ec/documentos/web-inec/Bibliotecas/Libros/Trabajo_Infantil_en_Ecuador.pdf

Migraciones, O. I. para las. (2015). Los migrantes y las ciudades: Nuevas colaboraciones para gestionar la movilidad Organización Internacional para las Migraciones (OIM) (June J.H. Lee (ed.)). www.iom.int

Ministerio de Educación del Ecuador. (2015). Modelo del Sistema de Educación Intercultural Bilingüe (pp. 1-27). Subsecretaría de Educación Intercultural Bilingüe. https://educacion.gob.ec/wp-content/uploads/ downloads/2014/03/MOSEIB.pdf

Ministerio de Salud Pública del Ecuador. (2018). Manual del Modelo de Atención Integral de Salud - MAIS.

Observatorio de Desplazamiento Interno (IDMC). (n.d.). Informe Mundial sobre Desplazamiento Interno 2020 (Vol. 24). Retrieved January 26, 2021, from https://www.internal-displacement.org/sites/default/ files/inline-files/GRID_Global_2020_Spanish_web. pdf $\#$ page $=61 ? \mathrm{v}=0.2$

Organización Mundial de la salud. (2021). OMS Constitución. https://www.who.int/es/about/who-we-are/ constitution

Pontificia Universidad Católica. (2019). Proyecto Académico PUCE. https://www.flipbookpdf.net/web/files/ uploads/6190cedf0a801444d81b474854d3c2c36a6624 82FBP21791591.pdf

Rodríguez Cruz, M. (2018). Construir la interculturalidad. Políticas educativas, diversidad cultural y desigualdad en el Ecuador. Íconos - Revista de Ciencias Sociales, 60(60), 217. https://doi.org/10.17141/iconos.60.2018.2922

Schoenfeld, P. G. (2010). Inclusión de la variable etnia en las fuentes de información sociodemográfica del Ecuador.

“Tránsito Amaguaña," U. E. I. bilingue. (2019). Proyecto educativo institucional. https://ueibtransitoamaguana.es.tl/ 